Hartmann, Nachtrag über persistirende Vasa omphalomesaraica. 163

Tag ihrer Menses am 13. October 1867, - am 16. October Coitus, Geburt am 11. Juli 1868, also wiederum 269 Tage nach der Empfängniss.

Beide Kinder waren reif und gesund, das erste ein Mädchen, das zweite ein Knabe. Auf sämmtliche obige Daten ist genau geachtet worden, der Beischlaf ward nicht an einem dem genannten nahen Tage ausgeübt, und die Angaben sind absolut zuverlässig.

Wahrscheinlich hat eine erneute Schwangerschaft derselben Frau wieder eine ganz gleiche Dauer gehabt, doch ist der Tag der Befruchtung nicht mit derselben Sicherheit anzugeben. Am 29. Januar 1869 traten die Menses ein, acht Tage nach dem Aufhören derselben hat ein Beischlaf stattgefunden, die Geburt eines reifen Knaben erfolgte am 5. November 1869. Wenn nun die befruchtende Cohabitation am 9. März, zwölf Tage nach dem Beginne der Menstruation, ausgeführt ward, was wahrscheinlich ist, so hat auch diese Schwangerschaft (die zehnte, mit Einschluss von zwei nicht vollendeten) 269 Tage gedauert.

\title{
Nachtrag über persistirende Vasa omphalomesaraica
}

ron

Dr. G. Hartmann in Stuttgart.

Als nachträglichen Zusatz zu meinen Beobachtungen über die oben genannten Gebilde (siehe Monatsschrift f. Geburtskunde Band XXXIII. S. 193) möchte ich mir zwei Bemerkungen erlauben: über die Häufigkeit des Vorkommens nämlich und über einen früheren Beobachter derselben.

Was zunächst ihre Häufigkeit anlangt, so* habe ich nummehr unter circa 800 Nachgeburten neun Fälle gefunden, was, wenn ich auch die beiden ersten als ganz zufällige Erfunde weglasse, immer noch einer Frequenz von beinahe 1 Proc. entspricht, so dass es eigenthümlich erscheint, dass nicht auch anderen Forschern diese Gebilde häufiger aufstossen. Zu einiger Erklärung dieser Factums kann ich übrigens beifügen, dass es zur Auffindung der genannten Gefässe ganz unumgänglich nothwendig ist, die Nachgeburten ganz frisch zu untersuchen, da durch die allgemeine blutige Imbibition der Eihuillen, 
164. Weber, Eine cystische Sacralgeschwulst als Geburtshinderniss.

die durch das Aufbewahren der Placenten im Wasser, oder auch ohne dasselbe, in ganz kurzer Zeit vor sich geht, die feinen Gebilde nahezu unsichtbar werden.

Bezutglich des zweiten Punktes habe ich bei Meckel ${ }^{1}$ ) in dem Kapitel über Anomalien der Placenta, ein Citat aus Kerkring ${ }^{2}$ ) gefunden über einen aus sieben Lappen bestehenden Mutterknchen, zu dem Meckel wörtlich bemerkt: „In dem Kerkring' schen Falle "ist es höchst merkwürdig und für das Wesen der Missbildung spre",chend, dass die Nabelgekrösgefässe sich erthalten haben."

Durch die Güte des Herm Prof. Luschka bekam ich auch das Kerkring'sehe Original zur Hand, dessen Wortlant, soweit er uns interessirt, ich hier folgen lasse: „Puer autem, qui huic placentae "(die aus sieben Lappen bestand) adjacebat hoc etiam praeter mo„rem aliorum ostendebat, venarum umbilicalium unam ex hepate, „prout fieri amat, alteram vero, quod a more abhorset, ex vena me, saraica prodeuntem. Arteriae item umbilicales, non ab extremitate "zortae, nec ramis iliacis sumebant initium, sed dnobus transversis "digitis ab aortae extremitate."

Dieser Passus hat allerdings meine durch Meckel's Worte hochgespannte Erwartung ziemlich herabgedrückt, und derselbe ist jedenfalls verschiedener Deutung fähig; einer Mittheilung hielt ich ihn aber dennoch für würdig.

\section{Eine cystische Sacralgeschwulst als Geburtshinderniss.}

$$
\text { Von }
$$

Dr. C. Weber in Mainz.

Frau M., 35 Jahre alt, seither vollständig gesund, Mutter von drei kräftigen Kindern, fing am Morgen des 27. September 1869, nach einer völlig normalen Sehwangerschaft an zu kreissen. Nachmittags gegen 4 Uhr ging das reichliche Wasser ab; gegen 8 Uhr Abends, nachdem der Kopf schon sehr herabgetreten, wurden die

1) Meckel, Handbuch der patholog. Anatomie. 1812. I. S. 88 .

2) Th. Kerkringi opera omnia anatomica. Lugduni Batav. 1727. Observatio XXXVII.: Placenta mulieris septem codyledonibus constans. 